\title{
Complete remission of multiple liver metastases with only partial response of the primary rectal cancer after neoadjuvant chemotherapy
}

\author{
Satomi Miura ${ }^{1}$, Kyoji Ito ${ }^{1}$, Nobuyuki Takemura ${ }^{1 *} \mathbb{D}$, Fuminori Mihara ${ }^{1}$, Tomomichi Kiyomatsu ${ }^{2}$ and Norihiro Kokudo ${ }^{1}$
}

\begin{abstract}
Background: Colorectal cancer is commonly diagnosed among the Japanese population, and various strategies in treating the colorectal liver metastasis have been introduced over the years. Here, we present a case of colorectal liver metastases in which we devised a multidisciplinary treatment plan for a better prognosis.

Case presentation: We report a case of a 44-year-old female who developed rectal cancer with advanced synchronous liver metastases and was treated by a liver-first surgical approach following neoadjuvant chemotherapy. At diagnosis, there were 12 bilobular lesions in the liver, and the primary rectal cancer was asymptomatic and unprogressive. We adopted a liver-first strategy because the control of the liver metastases was considered the key prognostic factor. Furthermore, because the lesions were highly progressive, we planned neoadjuvant systemic chemotherapy first to provide an observational period to identify potential new metastatic lesions that were refractory to systemic chemotherapy or contraindicative for surgical resection. We administered two courses of S-1 + oxaliplatin (SOX)+ bevacizumab (BV) and an additional course of SOX without BV as neoadjuvant chemotherapy in preparation for surgery. This resulted in a prominent minimalization of colorectal liver metastases, and no other remote metastasis was observed. Then, surgical resection of the colorectal liver metastases was performed safely, and the pathological result revealed complete remission of all tumors by neoadjuvant chemotherapy. The primary tumor in the colon was successfully resected 2 months after the hepatectomy. Although the patient experienced a recurrence in two different sites in the lungs 10 months after resection of the primary rectal lesion, these metastases were successfully resected after diagnosis. The patient is alive with no signs of recurrence 3 years after the diagnosis of colorectal cancer with synchronous liver metastases.

Conclusions: The combination of a liver-first strategy and neoadjuvant chemotherapy is a possible treatment of choice to cure colorectal cancer with simultaneous advanced colorectal liver metastases.
\end{abstract}

Keywords: Colorectal liver metastases, Neoadjuvant chemotherapy, Liver-first strategy

\section{Background}

Globally, colorectal cancer (CRC) is the third most commonly diagnosed cancer among men and second among women. This is mirrored in the Japanese population [1]. The prognosis of CRC has improved over the recent years; stage IV colon cancer with distant metastasis is no

\footnotetext{
* Correspondence: ntakemura@hosp.ncgm.go.jp

${ }^{1}$ Hepato-Biliary-Pancreatic Surgery Division, Department of Surgery, National Center for Global Health and Medicine, 1-21-1 Toyama, Shinjuku-ku, Tokyo 162-8655, Japan

Full list of author information is available at the end of the article
}

longer considered incurable [2]. In particular, liver metastasis is one of the most common metastatic sites of colorectal cancer, and to date, surgical and chemotherapeutic approaches have been shown to improve the overall survival of the patients with colorectal liver metastases (CRLM) [2]. However, the treatment strategy for colorectal cancer with synchronous liver metastases remains to be an important issue. The order in which surgery should be performed (liver-first, colorectal-first, or 
simultaneous) and the efficacy of neoadjuvant chemotherapy are provoking controversy around the world [3].

In this article, we presented a case of colorectal cancer with advanced synchronous liver metastases which were treated by a combination of neoadjuvant chemotherapy and a liver-first surgical approach.

\section{Case report}

A 44-year-old woman was identified as having positive fecal occult blood and a halo within the left side of the liver upon ultrasonography at a regular medical checkup and was referred to our hospital in November 2017. A colonoscopy revealed a type 2 tumor in the rectum (Fig. 1a), and an adenocarcinoma was detected by biopsy from the tumor. Contrast-enhanced computed tomography (CT) showed a well-defined solid mass in the lower rectum (Fig. 1b) and bilateral and multiple (12 in total) low-attenuation lesions in the liver (Fig. 1c, d). The patient was diagnosed with rectal cancer with synchronous advanced liver metastases. Additional remote metastases were not detected, and therefore, surgical resection of the primary rectal cancer and liver metastasis was indicated. Due to the fact that the primary tumor in the rectum was small and the rectum was unobstructed, treatment for the CRLM was considered to be the key prognostic factor, and therefore, the liver-first strategy was adopted. At that stage, the indocyanine green retention rate after 15 min (ICG-R15) was $11.4 \%$, and the estimated remnant liver volume was $61.1 \%$; therefore, a liver surgery was considerable. However, because the CRLM lesions were highly progressive, we planned a neoadjuvant systemic chemotherapy first to provide an observational period to identify potential new metastatic lesions, which were refractory to systemic chemotherapy or contraindicative for surgical resection. Two courses of $\mathrm{S}-1$ + oxaliplatin (SOX) + bevacizumab (BV) were administered (21-day cycle; day 1: oxaliplatin $\left(130 \mathrm{mg} / \mathrm{m}^{2}\right)$ + BV $(7.5 \mathrm{mg} / \mathrm{kg})$; day $1-14: \mathrm{S}-1\left(70 \mathrm{mg} / \mathrm{m}^{2} /\right.$ day $\left.)\right)$, and an additional course of SOX without BV was performed in preparation for surgery. At this point, the patient presented low white blood cell count (grade 2), generalized weakness (grade 2), loss of appetite (grade 1), and constipation (grade 1). The enforcement of chemotherapy resulted in a considerable reduction in the size of the tumor in both the rectum (Figs. 1a, b and 2a, b) and the liver (Figs. 1c, d and 2c, d). The primary tumor wall thickening was reduced from $20 \mathrm{~mm}$ to $13 \mathrm{~mm}$ on CT, and upon colonoscopy, the tumor was seen as a $20 \mathrm{~mm}$ size type 2 lesion before chemotherapy (Fig. 1a), whereas the tumor was prominently reduced to nothing but a flat a.

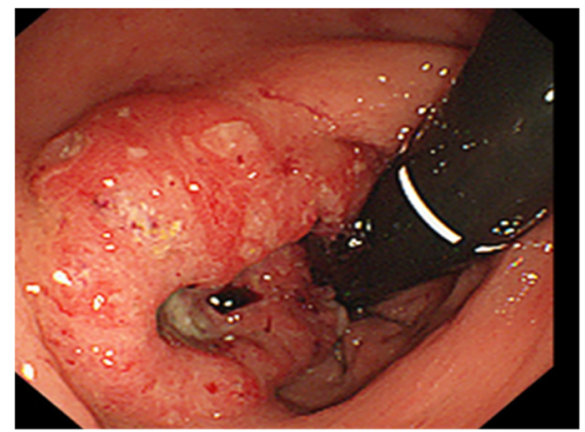

c.

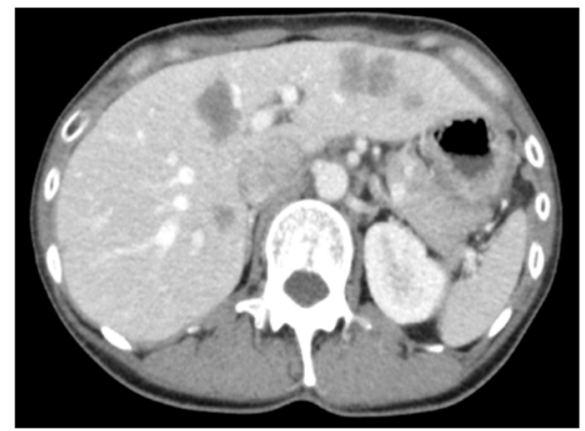

b.

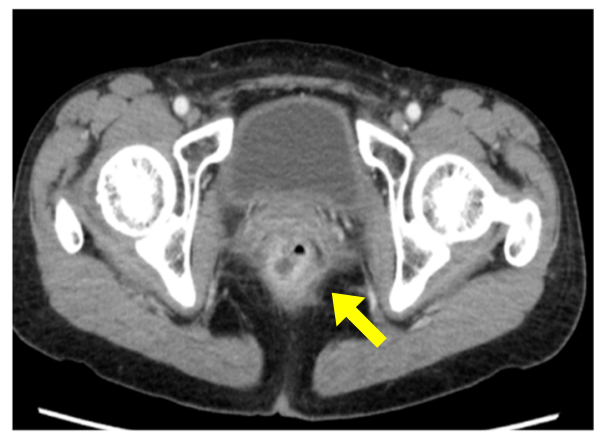

d.

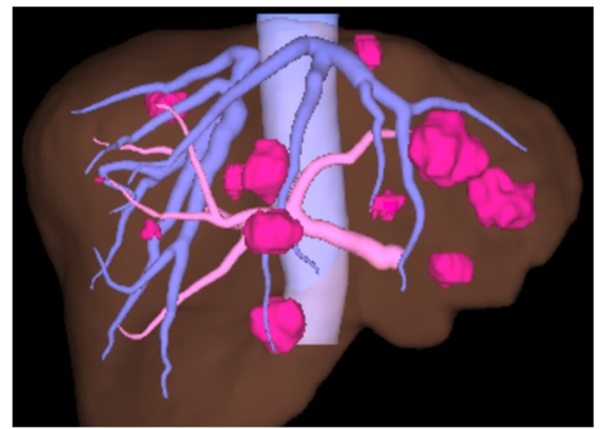

Fig. 1 CT imaging and a colonoscopy before neoadjuvant chemotherapy. a Primary rectal cancer on a colonoscopy. Type 2 rectal carcinoma. No bleeding or occlusion is observed. b Axial view of primary rectal cancer (arrow) on CT. Contrast-enhanced wall-thickening (20 mm) is seen in the lower rectum. There were no signs of occlusion. c Axial view of multiple bilobular liver metastases on CT. d Multiple liver metastases on 3dimentional image from $C T$. Lesions are bilobular. $C T$, computed tomography 
a.

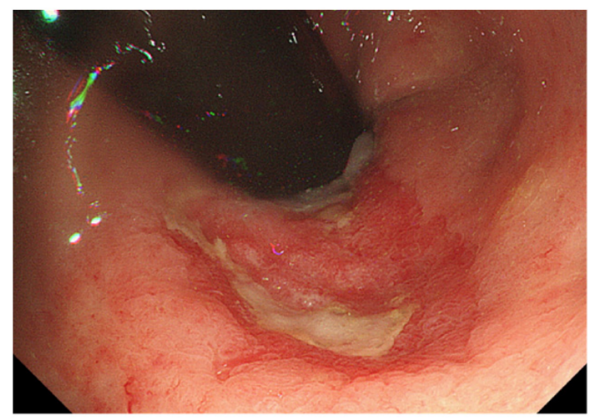

C.

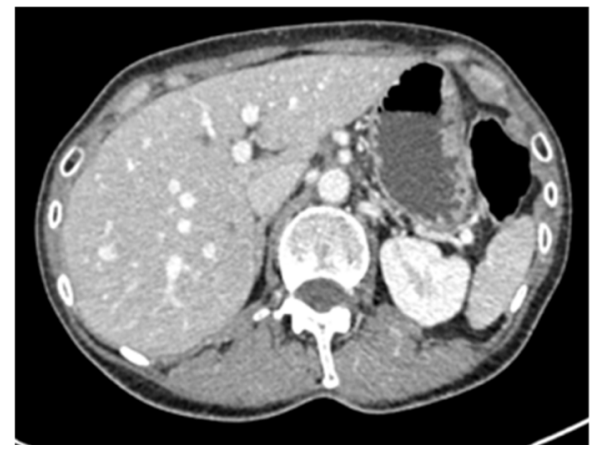

b.

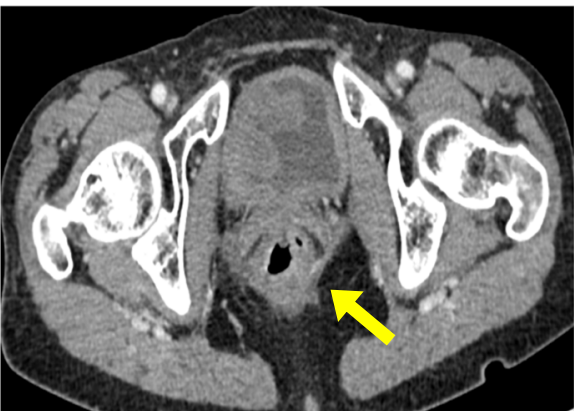

d.

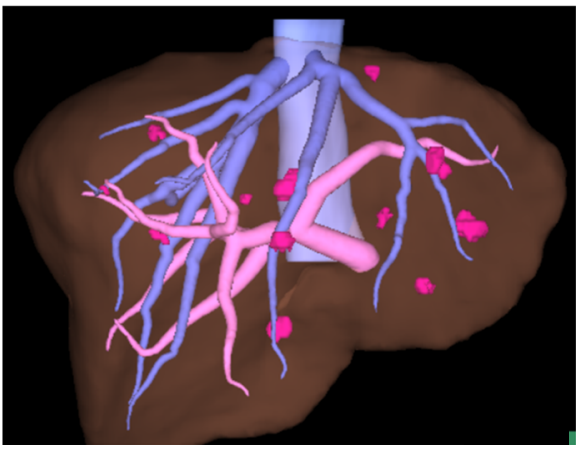

Fig. $\mathbf{2} \subset$ imaging and a colonoscopy after neoadjuvant chemotherapy. a Primary rectal cancer on a colonoscopy. The rectal cancer became flat and exceedingly shrunken. $\mathbf{b}$ Axial view of the primary CRC (arrow) on a CT. Contrast-enhanced wall-thickening $(13 \mathrm{~mm})$ has been improved. $\mathbf{c}$ Axial view of multiple bilobular liver metastases on $C$. The metastatic lesions shrunk prominently. $\mathbf{d}$ The 3-dimensional image of the multiple bilobular liver metastases. All metastatic lesions shrunk prominently. The estimated remnant liver volume was $73.4 \%$. CRC, colorectal cancer; $C$, computed tomography

granulation scar after chemotherapy (Fig. 2a). In addition, the pathology upon colonoscopic biopsy detected nothing but inflammatory cells, and therefore we have concluded that both the primary and CRLM lesions responded well to chemotherapy. On the course of chemotherapy, there was no great change in the serum albumin, total bilirubin, or prothrombin time, and no ascites was observed. ICG-R15 was $12.0 \%$ even after chemotherapy. The estimated remnant liver volume was $73.4 \%$ at this point (Fig. 2d). Considering the fact that a.
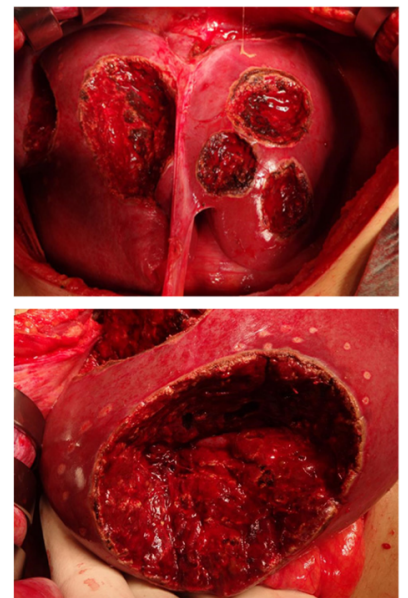

b.
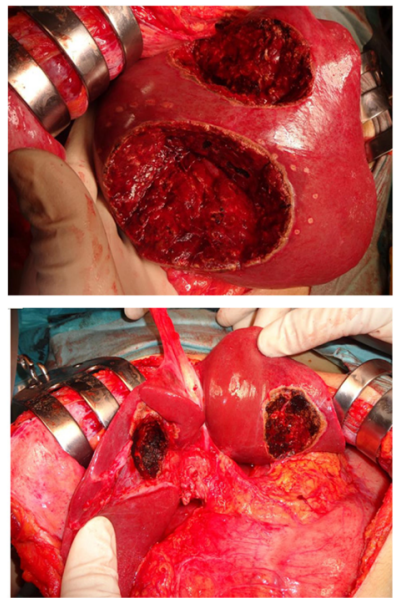

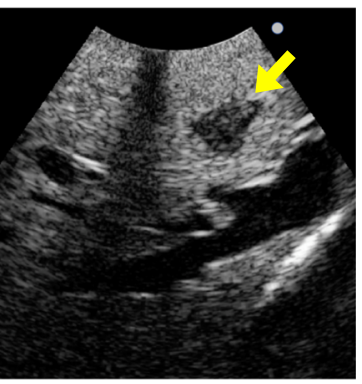

Fig. 3 Intraoperative images in CRLM resection. a Resection surface of the liver after a hepatectomy. $\mathbf{b}$ Contrast-enhanced ultrasonography using Sonazoid. Shrunken CRLM lesions were visualized as defects. CRLM, colorectal liver metastases 
the CRLMs were multiple and bilobular, the liver-first strategy was estimated to be suitable for the case. Eleven weeks after the introduction of neoadjuvant chemotherapy and 3 weeks after the final chemotherapy ( 8 weeks after the final administration of the final BV), a total of 10 partial hepatectomies were performed to extirpate 12 lesions. The clamp crushing method and the Pringle maneuver was adopted within the procedure (Fig. 3a). Because all tumors were located close to the liver surface, all lesions were accessed easily without division of main branches of the portal vein or hepatic vein. We used a contrast-enhanced ultrasonography by Sonazoid to detect the CRLM lesions, which were reduced after chemotherapy (Fig. 3b). The operative time was 7 h 12 $\mathrm{min}$, and the total blood loss was $1334 \mathrm{ml}$. The postoperative course was uneventful, and the patient was discharged from the hospital on postoperative day 9. The pathology of the surgical specimen revealed nothing but scars replacing what supposedly were lesions of liver metastasis. No residue of cancerous cells was observed within the scar; therefore, it was concluded that complete remission was achieved through chemotherapy (Fig. 4a, b). Pathology revealed no findings of edema, fibrosis, or thrombosis around the central hepatic vein and the sinusoids in the background liver, indicating the fact that there were no signs of sinusoidal obstruction syndrome. Two months after the hepatectomy, a laparoscopic intersphincteric resection of the primary rectal tumor was performed. The operative time was $6 \mathrm{~h} 7$ min, and the blood loss was $86 \mathrm{ml}$. The pathology showed a moderately differentiated adenocarcinoma penetrating into the muscularis propria. Most cancerous cells were viable (Fig. 4c, d). There was no lymph node metastasis. After further analysis, although the primary lesion seemed exceedingly diminished after neoadjuvant chemotherapy upon colonoscopy, the preoperative chemotherapy was defined as grade $1 \mathrm{a}$, and we concluded that its effect on the primary focus remained limited. The patient had a recurrence in two different sites in the lung (a single node in the lower-right lobe and another in the lower-left lobe) 10 months after the resection of the primary CRC lesion. A 2-stage surgery was performed to resect the metastatic lesions in the lungs. Three years after the diagnosis of colorectal cancer with synchronous liver metastases, there have been no signs of recurrence and the patient is still alive. The clinical time course of the patient and the change in the levels of tumor markers are summarized in Fig. 5.

\section{Discussions}

In the recent years, the prognosis of CRC has been improving [2]. Several treatment strategies are potentially key for improving the prognosis of patients with CRC. These include closer follow-up after resection and earlier detection of metastatic disease, improvement in chemotherapeutic regimens, and the enhancement of meetings held by multidisciplinary teams and tumor boards [4]. Typical metastatic lesions of colorectal cancer include a.

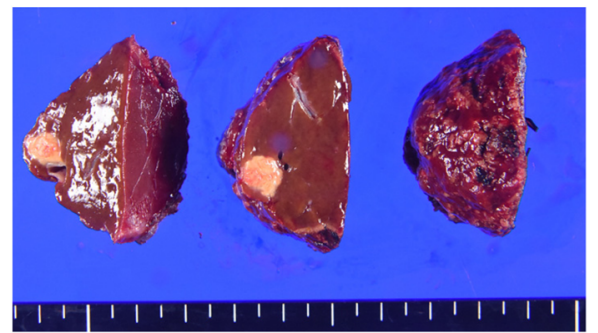

C.

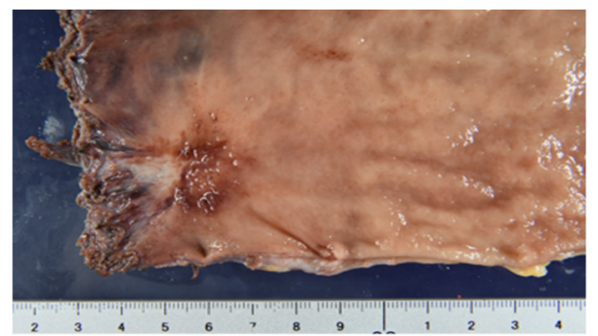

b.
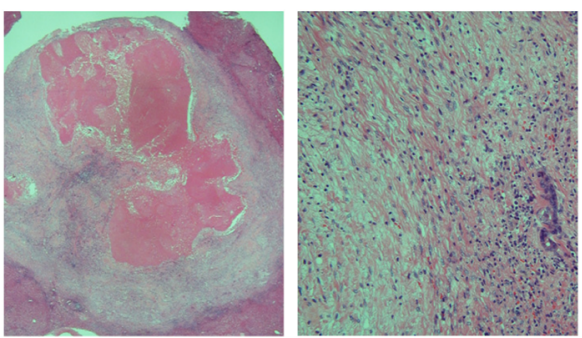

d.
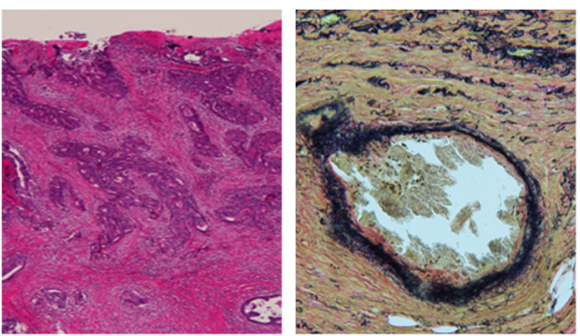

Fig. 4 Pathological findings of the specimen. a Macroscopic view of the liver metastases lesion (S4). $\mathbf{b}$ Distant and short-range view of hematoxylin and eosin staining of the liver metastases lesion. No viable carcinomic cells were identified. c Macroscopic view of CRC lesion. $\mathbf{d}$ Distant view of hematoxylin and eosin staining (left) and short-range view of Elastica van Gieson staining (right) of the CRC lesion. Glandular structure of intermediate-differential adenocarcinoma and viable carcinomic cells were packed within a small vein. CRC, colorectal cancer 


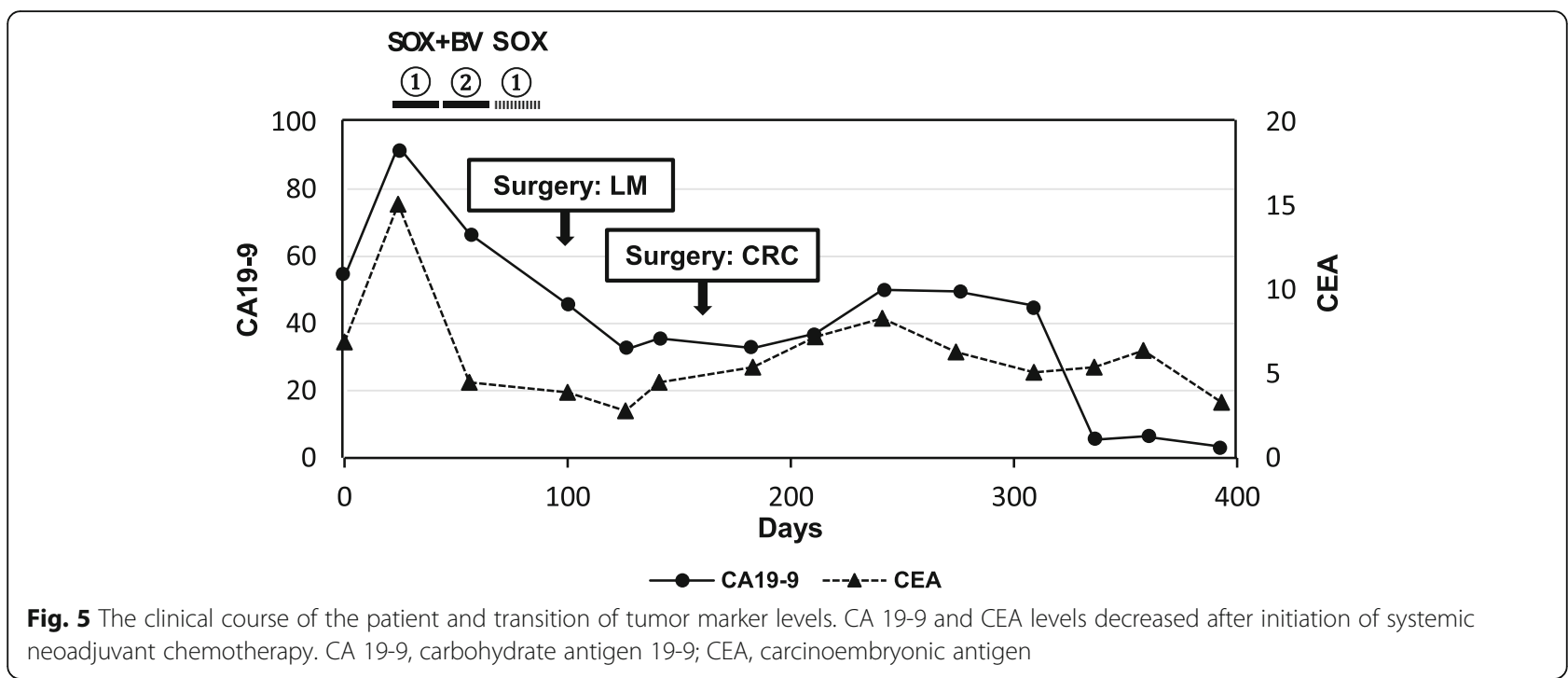

the liver, lung, peritoneum, nodes, and ovary, with the liver being the most common site of metastasis overall [5].

In CRLM treatment, hepatic resection remains to be the only curative option. If macroscopic surgery is determined to be feasible and if the maintenance of at least $30 \%$ of future liver remnant may be sustained under R0 resection, surgery should be the first-line therapy [2]. If the patient has no known underlying illnesses, no prognostic factor will overturn the choice of treatment [6]. However, there is no standard approach with regard to the order of surgical resection for synchronous liver metastases of CRC. There are no differences reported among the colorectal-first (classic), liver-first (reverse), and simultaneous approaches [3]. Generally, if the patient is presenting with symptoms such as bleeding, obstruction, and/or perforation, CRC resection should be performed first followed by systemic chemotherapy and the resection of CRLM [7]. If the CRC lesion is asymptomatic, a liver-first strategy may be an option to prevent chemotherapy-induced liver damage before hepatectomy and progression of the CRLM lesions [8]. If the colorectal lesion is uncomplicated and the number of liver metastases is limited, simultaneous resection of all lesions may be chosen [9]. With any approach, it is important to consider the treatment plan independently for each case based on the characteristics of the CRC and CRLM through close observation of imaging modalities [10]. In the present case, the rectal lesion was asymptomatic and unprogressive, and the liver lesion was highly progressive. Therefore, CRLM was clearly considered to be a primary prognostic factor, and we adopted a liver-first strategy for the present case.

Although hepatic resection remains the only curative option for CRLM, long-term survival or curing the condition is reported to be realized in only $20-50 \%$ of patients who have undergone R0 resection of CRLM [11]. The meta-analysis reported by Kansas GP et al. demonstrated that approximately half of the patients with resectable liver-limited CRC metastases developed widespread systemic disease within 3 years of resection [12]. Although systemic chemotherapy is an alternative treatment option for CRLM, there is no single consensus for the peri-operative chemotherapy to CRLM, and the best criteria for perioperative chemotherapy is yet to be decided. In the ESMO guideline, published in 2016, for patients with unresectable CRLM who may withstand intensive therapy, an upfront active combination regimen is recommended [2]. Generally, if the patient has a good performance status with five or more CRLMs, portal node involvement, or bilobular CRLM lesions are noted, neoadjuvant chemotherapy is recommended before surgical resection. Neoadjuvant chemotherapy is effective in many ways; it helps to see whether surgical treatment is possible or not in terms of the identification of potential new metastatic lesions which were contraindicated for surgery and also prevents systemic progression of the tumor [13]. However, it is also reported that downstaging is only attained in $10-15 \%$ of patients who undergo neoadjuvant chemotherapy [14]. In the present case, the patient was young and presented with a good performance status at diagnosis, and the liver lesion was highly advanced presenting bilobular spread and 12 tumor lesions. Therefore, the patient was considered to be a suitable case for neoadjuvant chemotherapy to secure an observational period to confirm that there was no other distant metastasis, except CRLM.

Chemotherapy must be planned accounting for the patient's liver function. Chemotherapy for over 16 weeks before surgical resection increases the risk of liver injury 
and post-operative complications, without pathologic improvement [15]. Long-term chemotherapy may lead to liver toxicity which may appear as symptoms, such as steatohepatitis [16] and portal hypertension [17]. In our case, the patient had a satisfactory liver function, and no obvious liver damage was seen along the course of chemotherapy.

Adam et al. reported an analysis of CRLM patients who underwent surgery in their institution, concluding that complete remission may occur in almost one-third of objective responders 60 years or younger with metastases $\leq 3 \mathrm{~cm}$ and with low carcinoembryonic antigen (CEA) values [18]. Our case met all of these conditions, which was a clear indication for the conduction of neoadjuvant chemotherapy, which resulted in a prominent minimalization of the CRLMs upon imaging. Surgical resection was then performed safely, and the pathological result revealed complete remission of all CRLMs by neoadjuvant chemotherapy. The primary tumor in the colon was successfully resected 2 months after CRLM resection. Therefore, neoadjuvant chemotherapy was clearly effective for this patient, and the liver-first strategy plus neoadjuvant chemotherapy could be a treatment choice to cure CRC with simultaneous advanced CRLM.

In our case, both the primary and the metastatic sites seemed to have a great sensitivity to neoadjuvant chemotherapy upon CT and colonoscopy. However, final pathological findings of the primary lesion revealed viable cells existing adjacent to the muscularis propria with vascular invasion, while the pathology for the liver lesions presented complete remission. Gervaz et al. compared histological response in colorectal liver metastases and the primary lesion in 29 patients, in which all were treated with a neoadjuvant chemotherapy followed by liver-first surgery, and then the primary lesion surgery [19]. There was a significant difference in the complete absence or significantly poor tumor response to chemotherapy between primary tumors (35.7\%) and liver metastases (6.9\%). Gervaz et al. hypothesized that a better drug delivery to the liver and the therapeutic interval of the primary lesion after CRLM resection causing repopulation of cancer cells may be a cause of worse tumor response in the primary lesion compared to the CRLMs. In our case, the surgery for the primary tumor was performed approximately 2 months after the resection of CRLMs, which could also affect the difference in the effectiveness of the neoadjuvant chemotherapy between the primary lesion and CRLMs.

In conclusion, neoadjuvant chemotherapy and a liverfirst surgical approach could be a possible curable treatment for asymptomatic colon cancer with simultaneous advanced CRLM, especially for young patients with no rise in CEA and with no CRLM lesions over $3 \mathrm{~cm}$ in size. Neoadjuvant chemotherapy played a key role in treating unresectable, but potentially curable, metastatic CRC.

\section{Abbreviations}

BV: Bevacizumab; CEA: Carcinoembryonic antigen.; CRC: Colorectal cancer; CRLM: Colorectal liver metastases; CT: Computed tomography; ICGR15: Indocyanine green retention rate after 15 min; SOX: S-1 + oxaliplatin

\section{Acknowledgements}

None.

\section{Authors' contributions}

$\mathrm{SM}, \mathrm{KI}$, and NT contributed to the conception and design of the study. All authors contributed to the acquisition and analysis of data. S.M, Kl, and NT were major contributors in writing the manuscript. All authors read and approved the final manuscript.

\section{Funding}

No funding was obtained from the private or public sector for this research.

\section{Availability of data and materials}

The datasets analyzed during the current study are not publicly available due to their containing information that could compromise the privacy of research participants but are available from the corresponding author on reasonable request.

\section{Ethics approval and consent to participate}

This article satisfied the consensus of the National Center for Global Health and Medicine Research Ethics Committee/Institutional Review Board.

\section{Consent for publication}

Informed consent was obtained from the patient presented in this article.

\section{Competing interests}

The authors declare that they have no competing interests.

\section{Author details}

${ }^{1}$ Hepato-Biliary-Pancreatic Surgery Division, Department of Surgery, National Center for Global Health and Medicine, 1-21-1 Toyama, Shinjuku-ku, Tokyo 162-8655, Japan. ${ }^{2}$ Colorectal Surgery Division, Department of Surgery, National Center for Global Health and Medicine, 1-21-1 Toyama, Shinjuku-ku, Tokyo 162-8655, Japan.

Received: 13 November 2019 Accepted: 17 February 2020

Published online: 27 February 2020

\section{References}

1. Doe J, Summary of updated cancer statistics in Japan. In: Updated cancer statistics. Center for Cancer Control and Information Services. National Cancer Center. 2019. https://ganjoho.jp/reg_stat/statistics/stat/summary. html. .

2. Van Cutsem E, Cervantes A, Adam R, Sobrero A, Van Krieken J, Aderka D, et al. ESMO consensus guidelines for the management of patients with metastatic colorectal cancer. Annals of Oncology. 2016;27(8):1386-422.

3. Kelly M, Spolverato G, Le G, Mavros M, Doyle F, Pawlik T, et al. Synchronous colorectal liver metastasis: a network meta-analysis review comparing classical, combined, and liver-first surgical strategies. J Surg Oncol. 2015; 111(3):341-51.

4. Shah S, Arora S, Atkin G, Glynne-Jones R, Mathur P, Darzi A, et al. Decisionmaking in colorectal cancer tumor board meetings: results of a prospective observational assessment. Surg endosc. 2014;28(10):2783-8.

5. Manfredi S, Lepage C, Hatem C, Coatmeur O, Faivre J, Bouvier A-M. Epidemiology and management of liver metastases from colorectal cancer. Ann surg. 2006;244(2):254.

6. Berri RN, Abdalla EK. Curable metastatic colorectal cancer: recommended paradigms. Curr Oncol Rep. 2009;11(3):200-8.

7. Poultsides GA, Servais EL, Saltz LB, Patil S, Kemeny NE, Guillem JG, et al. Outcome of primary tumor in patients with synchronous stage IV colorectal cancer receiving combination chemotherapy without surgery as initial treatment. J Clin Oncol. 2009;27(20):3379. 
8. Mentha G, Majno P, Andres A, Rubbia-Brandt L, Morel P, Roth A Neoadjuvant chemotherapy and resection of advanced synchronous liver metastases before treatment of the colorectal primary. Br J Surg. 2006;93(7): 872-8.

9. Lyass S, Zamir G, Matot I, Goitein D, Eid A, Jurim O. Combined colon and hepatic resection for synchronous colorectal liver metastases. J surg oncol. 2001;78(1):17-21.

10. Adam R, De Gramont A, Figueras J, Guthrie A, Kokudo N, Kunstlinger F, et al. The oncosurgery approach to managing liver metastases from colorectal cancer: a multidisciplinary international consensus. Oncologist. 2012;17(10): 1225-39.

11. Weiser MR, Jarnagin WR, Saltz LB. Colorectal cancer patients with oligometastatic liver disease: what is the optimal approach. Oncology. 2013; 27(11):1074-8.

12. Kanas GP, Taylor A, Primrose JN, Langeberg WJ, Kelsh MA, Mowat FS, et al. Survival after liver resection in metastatic colorectal cancer: review and meta-analysis of prognostic factors. Clin epidemiol. 2012;4:283.

13. Adam R, Delvart V, Pascal G, Valeanu A, Castaing D, Azoulay D, et al. Rescue surgery for unresectable colorectal liver metastases downstaged by chemotherapy: a model to predict long-term survival. Ann surg. 2004;240(4): 644.

14. Masi G, Loupakis F, Pollina L, Vasile E, Cupini S, Ricci S, et al. Long-term outcome of initially unresectable metastatic colorectal cancer patients treated with 5-fluorouracil/leucovorin, oxaliplatin, and irinotecan (FOLFOXIRI) followed by radical surgery of metastases. Ann surg. 2009;249(3):420-5.

15. Kishi Y, Zorzi D, Contreras CM, Maru DM, Kopetz S, Ribero D, et al. Extended preoperative chemotherapy does not improve pathologic response and increases postoperative liver insufficiency after hepatic resection for colorectal liver metastases. Ann surg oncol. 2010;17(11):2870-6.

16. Kooby DA, Fong Y, Suriawinata A, Gonen M, Allen PJ, Klimstra DS, et al. Impact of steatosis on perioperative outcome following hepatic resection. J Gastrointestinal Surg. 2003;7(8):1034-44.

17. Hubert C, Sempoux C, Horsmans Y, Rahier J, Humblet Y, Machiels JP, et al. Nodular regenerative hyperplasia: a deleterious consequence of chemotherapy for colorectal liver metastases? Liver International. 2007;27(7): 938-43

18. Adam R, Wicherts DA, de Haas RJ, Aloia T, Lévi F, Paule B, et al. Complete pathologic response after preoperative chemotherapy for colorectal liver metastases: myth or reality? J Clin Oncol. 2008;26(10):1635-41.

19. Gervaz P, Rubbia-Brandt L, Andres A, Majno P, Roth A, Morel P, et al. Neoadjuvant chemotherapy in patients with stage IV colorectal cancer: a comparison of histological response in liver metastases, primary tumors, and regional lymph nodes. Ann Surg Oncol. 2010;17(10):2714-9.

\section{Publisher's Note}

Springer Nature remains neutral with regard to jurisdictional claims in published maps and institutional affiliations.

\section{Submit your manuscript to a SpringerOpen ${ }^{\circ}$ journal and benefit from:}

- Convenient online submission

- Rigorous peer review

- Open access: articles freely available online

- High visibility within the field

- Retaining the copyright to your article

Submit your next manuscript at $\boldsymbol{\nabla}$ springeropen.com 